\title{
Phytotoxicological Assessment of Two Wetlands in Eloor, Kochi Using Aquatic Macrophyte Spirodela Polyrhiza.
}

\author{
${ }^{1}$ Anil Loveson and ${ }^{2}$ Dr. Rajathy Sivalingam \\ School of Environmental Studies, Cusat, Kochi-682022, Kerala, India \\ ${ }^{1}$ Anil Loveson, School of Environmental Studies, Cochin University of Science and Technology, Kerala, India. \\ ${ }^{2}$ Dr. Rajathy Sivalingam, Reader, School of Environmental Studies, Cochin University of Science and \\ Technology, Kerala, India.
}

\begin{abstract}
In the current study, the duckweed aquatic macrophyte Spirodela polyrrhiza was employed for assessing the toxicity of two wetlands in the Eloor industrial estate, Ernakulam district, Kerala, South India. The assessments were made according to OECD guidelines for testing (2006). The studies involve study of growth parameters, Growth Index, Biomass and changes in productivity. The water samples were collected from two different wetland sites at the same time. The spirodela plants were introduced into several dilutions of wetland water samples. The parameters were measured after 7 days of exposure. All samples except control affected all parameters. The results of this study emphasize the significance of duckweeds as standard and reliable testing material for biological parameters in polluted aquatic ecosystem.
\end{abstract}

Keywords: Growth inhibition, Spirodela polyrrhiza, Growth Index, macrophyte, duckweed

\section{Introduction:}

Wetlands support a wide array of flora and fauna and deliver many ecological, climatic and societal functions. Scientists often refer to wetlands as the" kidneys" of the earth. . Kerala is well known for its wetlands. Eloor, an island of $11.21 \mathrm{sq} / \mathrm{km}$, on the Periyar River is home to more than 247 chemical industries and large number of wetlands. The soil, water bodies and the wetlands in and around Eloor have been contaminated with heavy metals. Standardised ecotoxicity test methods frequently uses duckweed species Spirodela polyrrhiza due to their advantages such as rapid vegetative propagation, sensitivity to toxicants, easy culturing under axenic conditions ( Lakatos et al .1993).

\section{Materials and methods:}

Duckweed Spirodela polyrrhiza were obtained from an unpolluted natural pond near Fort Kochi, Kerala, India. It is a floating aquatic macrophyte belonging to the family Lemnaceae and can be found world wide on the surface of fresh and brackish waters (Zimmo, 2003). The Lemna spp.are among the most standardized test organisms in aquatic ecotoxicology( EPA 1996;DIN2000,2001;Eberius 2001;OECD 2002). One sample (Wetland 1) was collected approximately 8 metres north of the Kuzhikundam Thodu creek, at a location approximately $10 \mathrm{~m}$ northwest of the HIL site boundary ( Lat $10^{\circ} 04^{\prime} 51.76$ ' $\mathrm{N}$ and Long $76^{\circ}$ $\left.17^{\prime} 32.55^{\prime \prime} \mathrm{E}\right)$. (See table 1)

The second sample ( Wetland 2) was collected from the wetlands southwest of the "Amanthuruthu" wetland area, approximately 150 metres west of the HIL ( Hindustan Insecticides Limited) site, and approximately 80 metres south of the Kuzhikundam Thodu creek ( Lat $10^{\circ} 04^{\prime} 48.13^{\prime \prime} \mathrm{N}$ Long $76^{\circ} 17^{\prime} 22.75^{\prime \prime} \mathrm{E}$ ) (see Table 2).

Test solutions were prepared by diluting water samples of wetland 1 and 2 with distilled water. The solutions were prepared in $100 \%, 50 \%, 25 \%, 10 \%, 5 \%$ and $0.5 \%$ concentrations of wetland water plus a control and undergo seven days of exposure, plants were harvested, washed with double distilled water, blotted and used for the study of various parameters. The parameters include study of vegetative characters, growth parameters and study photosynthetic pigments. All the tests were conducted in six replicates.

\section{Study of growth parameters}

\section{Analysis of parameters:}

\section{A.Dry weight:}

All colonies are collected from each of the test vessels and rinsed with distilled or deionised water. They are blotted to remove excess water and then dried at $60{ }^{\circ} \mathrm{C}$ to a constant weight. Any root fragments should be included. The dry weight should be expressed to an accuracy of at least $0.1 \mathrm{mg}$. 


\section{B. Fresh weight:}

All colonies are transferred to pre-weighed plastic tubes with small $(1 \mathrm{~mm})$ holes in the rounded bottoms. The tubes are then centrifuged at $3000 \mathrm{rpm}$ for 10 minutes at room temperature. Tubes, containing the now dried colonies, are re-weighed and the fresh weight is calculated by subtracting the weight of the empty tube.

\section{1.c Dry weight-Fresh weight}

ratio can be determined from above estimations. The plant growth index was calculated as follows Growth Index $=$ Biomass $(\mathrm{t}=7$ days $)$

$$
\text { Biomass }(\mathrm{t}=0)
$$

\section{D. .Doubling time:}

To determine the doubling time $(T d)$ of frond number and adherence to this validity criterion by the study, the following formula is used with data obtained from the control vessels:

$\mathrm{Td}=\ln 2 / \mu$ Where $\mu$ is the average specific growth rate .

\section{1.e. Average specific growth rate:}

This response variable is calculated on the basis of changes in the logarithms of frond numbers, and in addition, on the basis of changes in the logarithms of another measurement parameter (total frond area, dry weight or fresh weight) over time (expressed per day) in the controls and each treatment group. It is sometimes referred to as relative growth rate. The average specific growth rate for a specific period is calculated as the logarithmic increase in the growth variables -frond numbers and one other measurement variable (total frond area, dry weight or fresh weight) - using the formula below for each replicate of control and treatments:

$\mu_{\mathrm{i}-\mathrm{j}}=\ln \left(\mathrm{N}_{\mathrm{j}}\right)-\ln \left(\mathrm{N}_{\mathrm{i}}\right)$

where:

- $\mu \mathrm{i}-\mathrm{j}$ : average specific growth rate from time $\mathrm{i}$ to $\mathrm{j}$

- $\mathrm{Ni}$ : measurement variable in the test or control vessel at time $\mathrm{i}$

- $\mathrm{Nj}$ : measurement variable in the test or control vessel at time $\mathrm{j}$

$-\mathrm{t}$ : time period from $\mathrm{i}$ to $\mathrm{j}$.

\section{1.f. Percentage of growth inhibition:}

Percent inhibition of growth rate (Ir) may then be calculated for each test concentration according to the following formula

$\% \mathrm{Ir}=(\mu \mathrm{C}-\mu \mathrm{T}) \times 100$

$$
\mu \mathrm{C}
$$

where:

- \% Ir : percent inhibition in average specific growth rate

- $\mu \mathrm{C}:$ mean value for $\mu$ in the control

$-\mu \mathrm{T}:$ mean value for $\mu$ in the treatment group

\section{2. .Estimation of photosynthetic pigments.}

The chlorophyll estimation is an important study parameter for the estimation of impact of pollution on photosynthetic activity. About $200 \mathrm{mg}$ of treated plants were weighed. This is taken in a mortar with $5 \mathrm{mlof} 90 \%$ acetone and $1 \mathrm{ml}$ of Magnesium carbonate. It is then ground thoroughly with pestle. This is then kept at $4^{\circ} \mathrm{C}$ for 4 hours for the pigments to elute. The solution is then centrifuged at $2500 \mathrm{rpm}$ for 15 minutes. The extract is then decanted to a volumetric flask and the volume is made up to $50 \mathrm{ml}$ with $90 \%$ acetone. The absorbance at 750 , 663, 645,510 and 480 were measured in the spectrophotometric analysis using Hitachi-U-2000 spectrophotometer.

\section{Statistical Analysis}

Analysis of variance for each test were conducted using STATISTICA software ( One way Anova). The significant difference between treatments were determined by Duncan's multiple range test $(\mathrm{P}<0.05)$. Each test was conducted in six replicates.

\section{Results and conclusions:}

The concept of average specific growth rate is based on the general exponential growth pattern of duckweed in non-limited cultures, where toxicity is estimated on the basis of the effects on the growth rate, without being dependent on the absolute level of the specific growth rate of the control; slope of the 
concentration-response curve or on test duration. The use of average specific growth rate for estimating toxicity is scientifically preferred. In the current study ASGR and frond doubling time (Td) of the control and treatment with $0.5 \%$ concentration yield the same result. The inhibition of growth in this concentration is negligible. As the concentration of effluent increases, all the parameters vary. When the frond doubling time exceeds 2.5 , the test solution is considered toxic. In the study 50\% and 100\% concentration treatments in wetland 1 and 2 shows Td values more than 2.5 , thus found to be toxic. The values are given in table 3 .

Plant growth index were measured after 7 days of exposure with different dilutions of water from both wetlands. At $0.5 \%$ and 5\% dilution GI is greater than control values in both waters. At $10 \% \mathrm{~W} 2$ treatment shows less GI than control but W1 still has values above control which shows the water quality of W1 is better than W2. From 25\% GI values shows sharp decline in both treatments. The finding is given in table 4 .

The growth indexes of both wetland water are similar in lowest concentration $(0.5 \%)$. From $5 \%$ dilution onwards it is quite clear that growth of Spirodela is affected more in wetland II. But at 25\% dilution GI is surprisingly similar. At extreme a concentration again GI varies. The differences in GI under different concentrations are illustrated in Table 3.

Changes in Dry weight fresh weight ratio indicate that in exposed Spirodela plants, growth retardation takes place in comparison to the control. At $0.5 \%$ concentration, the biomass yield is same as that in control. In $5 \%$ concentration of wetland 1and 2 water, slight increase in DW/FW ratio recorded in wetland 2 effluents than control. At 10\% and 25\% solutions, the DW/FW ratio is higher in weland water 1 in comparison with Wet 2. But in 50\% and $100 \%$ the ratio shows sharp decline. It was noted that at 50\% concentration, spirodela growing in highly polluted weland 2 water yield higher FW/DW ratio than those growing in wetland 1 ( Table 5). The decline in biomass ratio may be due the presence of excess heavy metals present in wetland. It has been shown that accumulation of heavymetals disturb the plant water status which eventually results in osmotic stress and growth reduction ( Perfus- Barbeoch et al.2002; Poschen-reider and Barcelo 2004). Water especially in wetland 2. In wetland 1 the biomass (DW/FW ratio) percentage were 100, 100.7,103.9, 100, 78.5, 64.27, and 57.1 for dilutions $0.5,5,10,25,50$ and 100 respectively. Similarly for wetland 2 it was $100,99.9,100.07,78.4,74.9$, 64.2 , and 57.04 for dilutions $0.5,5,10,25,50$ and 100 respectively.

Chlorophyll and carotenoids are the central part of the energy manifestation of every green plant system and therefore, any significant alteration in their levels is likely to cause a marked effect on the entire metabolism of the plant. The productivity of plants is directly related with changes in the content of photosynthetic pigments chlorophyll $\mathrm{a}$, chlorophyll $\mathrm{b}$ and Carotenoids. Industrial wastewater not only affects the chlorophyll content but the chlorophyll activity also(song and Huang,2001;Baron et al., 1995; Lewis, 1995). In the study photosynthetic pigments were inhibited due to metal toxicity. Duckweed leaves started to show signs of chlorosis (pigment loss) following $7 \mathrm{~d}$ exposure to surface water samples. At $50 \%$ and $100 \%$ dilutions necrosis could also seen. The Carotenoids contents are found to be less affected. At the end of 7 days of exposure $0.5 \%$ dilution of wetland 1 and 2 shows slight increase in chlorophyll a content (Hormesis) while at the same dilution and Carotenoids content remain unchanged. From 5\% to 100\% all pigments shows gradual decrease in concentration. $\mathrm{Chl} b$ degraded at a much slower rate than chl $a$ which indicates that greater damage of pollutants present in water samples on chl $a$.The loss of photosynthetic pigment content has been reported in duckweed plants following exposure to $\mathrm{Cu}, \mathrm{Pb}$ and $\mathrm{Ni}$ (Axtell et al.2003; Hou et al.2007; Kanoun- boule et al.2009).The destruction of photosynthetic pigments by heavy metals could be due to : impairment of ETC, replacement of Magnesium ions associated with chlorophyll ring, inhibition of important enzymes ( Van Assche and Clijsters 1990) associated with chlorophyll synthesis or peroxidation processes in chloroplast membrane lipids by reactive oxygen species ( Sandalio et al.2001).

\section{Acknowledgement:}

The authors are grateful to the School of Environment studies, CUSAT, for providing technical assistance to carry out the work.

\section{Conclusion:}

The study revealed that both welands are highly polluted. Wetland 2 has more pollutants compared with wetland 1 which is evident from the assessment of vegetative, growth and photosynthetic pigment parameters. The study also points towards the importance of conservation of wetlands in the area.

\section{References:}

[1] Axtell, N.R., Stenberg, S.P.K., Claussen, K.,(2003). Lead and Nickel removal using Microspora and Lemna minor, Bioresour. Technol.,89:41-48

[2] Baron,M.,J.B. Arellano and J.L.Gorge: Copper and photosystem II: a conversional relationship plant physiol.,94, 174-180(1995).

[3] DIN(2000) Duckweed growth Inhibition test: Determination of the non poisonous effect of water constituent $\mathrm{s}$ and waste water to duckweed, Deutsches Institut furcNormung e.V urchBeuth Verlag, Berlin, 19pp 
[4] Eberius M(2001) Assesment of Inhibition values and comprehensive analysis of biotests. LemnaTec GmbH,Wurselen, online http://www.lemnatec.com/Literatur/LT003.pdf

[5] EPA (1996) Aquatic plant toxicity test using LemnaSpp:Tiers I and II- OPPTS 850.4400.United states Environmental protection Agency Prevention, pesticides and Toxic substances Unit, Newyork,9 pp.

[6] Hou W, Chen X, SongG, Wang Q, Chang CC(2007) Effects of copper and cadmium on heavy metal polluted water restorationby Duckweed. Plant physiol Bioche 45:62-69

[7] Kanoun- Boule M. Vincente JAF, Nabais C, Prasad MNV, Freitas H(2009) Ecophysiological tolerance of duckweeds exposed to copper. Aquat Toxicol 91:1-9

[8] Lakatos et al(1993) Application of Lemna Species in ecotoxicological studies of Heavymetals; Sci Total Environ 44:773-778

[9] Lewis, M.A., Use of fresh water plant for phytotoxicity testing: A review. Environ.pollut.,87, 319-336(1995).

[10] OECD, (2002). Guidelines for the testing of chemicals. Lemna Sp. Growth Inhibition Test, Draft guidline 221

[11] Perfus- BarbeochL, Leonhardt N, Vavasseur A, Forestier C.(2002) Heavy metal toxicity: Cadmium permeates through calcium channels and disturbs the plant water status. Plant J 32:539-548.

[12] Poschenrieder C, Barcelo J (2004) Water relations in heavy metal stressed plants. In: Prasad MNV (ed) Heavy metal stress in plants: from biomolecules to ecosystems, $2^{\text {nd }}$ edn. Springer, Berlin,pp 249-270.

[13] Sandalio LM, Dalurzo HC, GomezM, Romero- Puertas MC, del Rio LA(2001)Cadmium-induced changes in the growth and oxidative metabolism of pea plants. J Exp Bot 52:2115-2126

[14] Song, Z.H., G.L. Huang: Effect of triphenyltin on duckweed Lemna minor. Bull. Environ.contam .Toxicol. 67, 368-375(2001).

[15] Van Assche F, Clijsters H(1990) Effects of metals on enzyme activity in plants. Plant cell Environ 13:195-206

[16] Zimmo, O. (2003). Nitrogen transformations and removal mechanisms in algal and duckweed waste stabilization ponds. PhD thesis, International Institute for Infrastructural, Hydraulic and Environmental Engineering, Delft, The Netherlands.

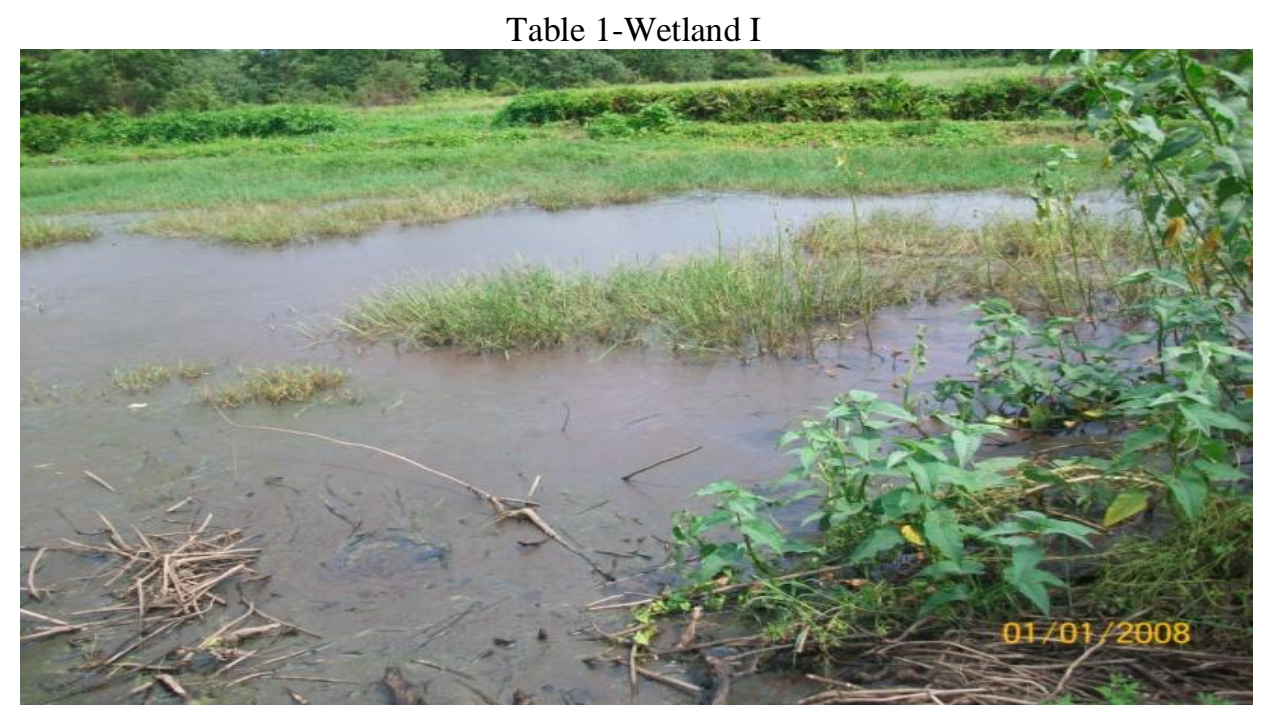

Table 2-Wetland II.

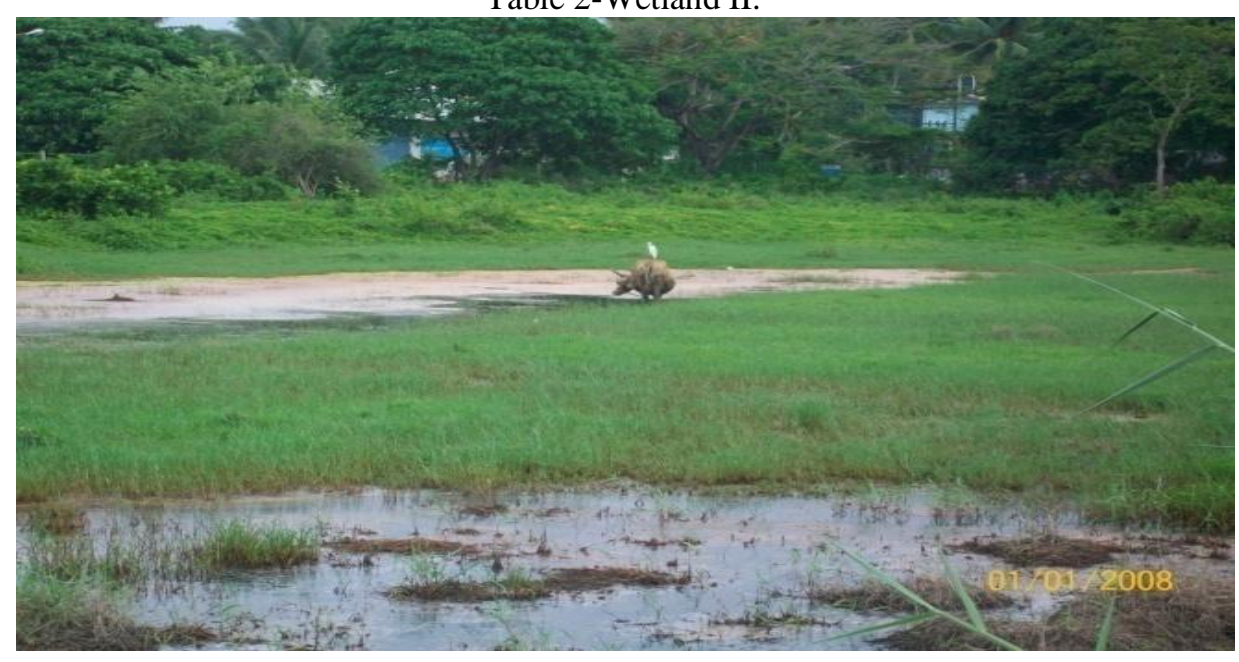

Table 3: ASGR, Td and Ir\% of S. polyrrhiza afer 7 days of treatment with various dilutions of wetland I and 2. Mean $N(i)=5$, Mean $T(j)=7$ and $T(i)=0$. Standard deviations were presented by error bars. Each values are means of sixreplicates. The significant difference between treatmentsis $P<0.05$. 


\begin{tabular}{|c|c|c|c|c|c|}
\hline \multirow[b]{2}{*}{ Site } & Medium & mean $\mathrm{Nj}$ & mean ASG ( $\boldsymbol{\mu})$ & Td & $\% \mathrm{Ir}$ \\
\hline & control & 16 & 4.258 & 1.272 & 0 \\
\hline \multirow[b]{6}{*}{ Wet I } & 0.5 & 16 & 4.258 & 1.272 & 0 \\
\hline & 5 & 15 & 3.871 & 1.4 & 9.08 \\
\hline & 10 & 14 & 3.484 & 1.555 & 18.177 \\
\hline & 25 & 12 & 2.71 & 2 & 36.35 \\
\hline & 50 & 10 & 1.935 & 2.801 & 34.21 \\
\hline & 100 & 9 & 1.548 & 3.501 & 63.64 \\
\hline \multirow[b]{6}{*}{ Wet 2} & 0.5 & 16 & 4.258 & 1.272 & 0 \\
\hline & 5 & 15 & 3.871 & 1.4 & 9.08 \\
\hline & 10 & 14 & 3.484 & 1.555 & 18.177 \\
\hline & 25 & 13 & 3.097 & 1.75 & 27.26 \\
\hline & 50 & 9 & 1.548 & 3.501 & 63.64 \\
\hline & 100 & 8 & 1.161 & 4.668 & 72.73 \\
\hline
\end{tabular}

Table 4: Growth Index of Spirodela plant in different dilutions of Wetland 1 and 2 after 7 days of exposure.Standard deviations were presented by error bars. Each values are means of sixreplicates. The significant difference between treatmentsis $P<0.05$.

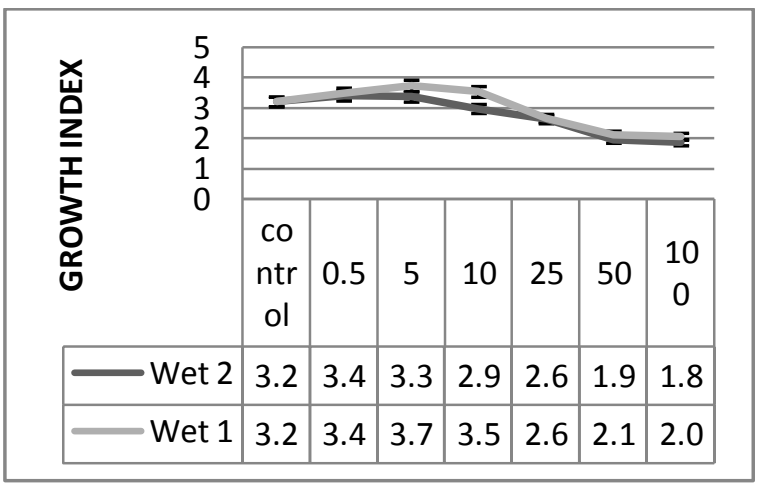

Table 5: Biomass DW/FW ratio of S. polyrrhiza after 7 days of exposure in water from Wetland 1 and 2. Standard deviations were presented by error bars. Each value is means of sixreplicates. The significant difference between treatmentsis $P<0.05$

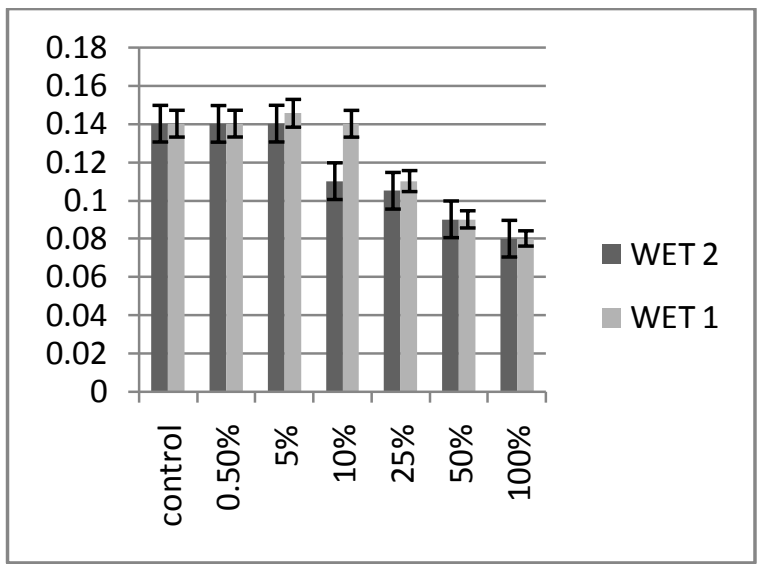

Table 6: Relative photosynthetic pigment concentrations after 7 days of exposure in different dilutions of wetland 1. Standard deviations were presented by error bars. Each values are means of sixreplicates. The significant difference between treatmentsis $P<0.05$. 


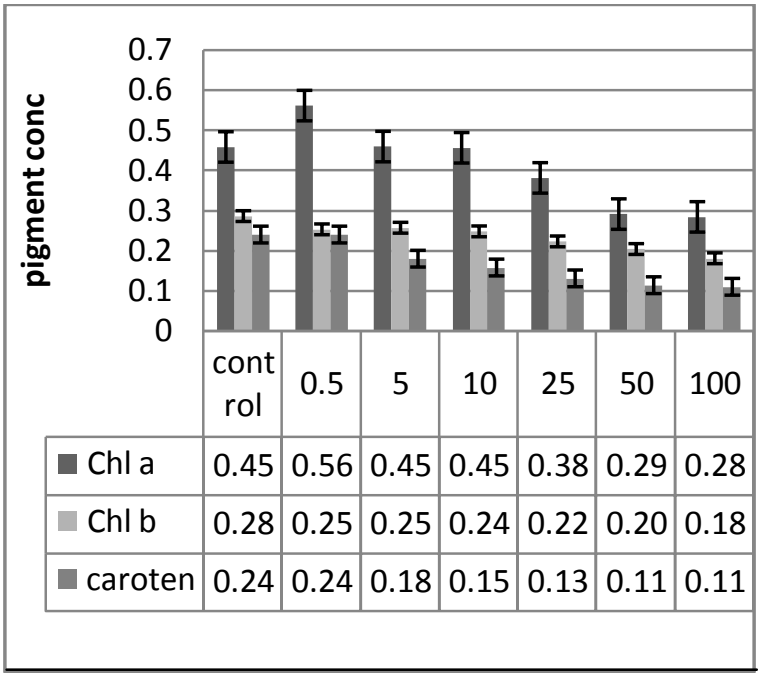

Table 7: Relative photosynthetic pigment concentrations after 7 days of exposure in different dilutions of wetland 2. Standard deviations were presented by error bars. Each values are means of sixreplicates. The significant difference between treatmentsis $P<0.05$.

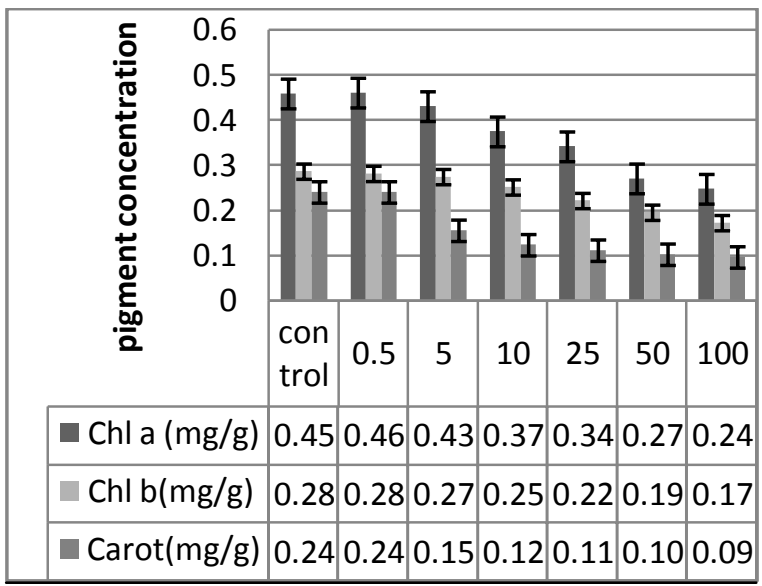

